\title{
Evolución cultural en sociedades artificiales
}

\section{Resumen}

En este artículo se revisan las generalidades de algunos modelos computacionales inspirados en características propias de la evolución cultural y se clasifican en dos grupos, según se fundamenten o no en la memética. Se pretende demostrar, a pesar del auge que han tenido en aplicaciones orientadas a solucionar problemas de optimización, su falta de naturalidad frente a la posibilidad de simular características culturales presentes en las sociedades naturales. La cultura se asume como el conjunto de ideas y comportamientos desarrollados y transmitidos mediante la interacción entre agentes portadores de genes y memes, pues se considera que estos tienen genéticamente codificada una maquinaria biológica con cierto sistema mental y que en este sistema están los memes capaces de comunicarse con otros agentes semejantes. Independientemente del hecho de tener neuronas en este sistema, en la mayoría de los modelos acá revisados no hay una relación genético-cultural de este tipo, pues en ellos lo cultural queda reducido a acelerar la resolución genética de problemas; o, en el mejor caso encontrado, lo cultural emplea memes derivados de los genes, pero como copias de su información génica.

Palabras clave: evolución cultural, genes, memes, memética, modelos computacionales, sociedades artificiales

\begin{abstract}
In this paper, the generalities of some computational models inspired by characteristics of cultural evolution are reviewed and classified into two groups, depending on whether they are based on memetics or not. It is intended to demonstrate, despite the boom they have had in applications aimed to solve optimization problems, their lack of naturalness against the possibility of simulating cultural characteristics present in natural societies. The culture is assumed as the set of ideas and behaviors developed and transmitted through the interaction between agents carrying genes and memes, since it is considered that they have genetically encoded a biological machinery with a certain mental system and that in this system are the memes capable of communicate with other similar agents. Regardless of the fact of having neurons in this system, in most of the models reviewed here there is no such genetic-cultural relationship, since in them the cultural is reduced to accelerate the genetic resolution of problems or, in the best case found, the cultural uses memes derived from genes, but as copies of their gene information.
\end{abstract}

Keywords: Artificial Societies, Computational Models, Cultural Evolution, Genes, Memes, Memetics

\footnotetext{
* Ingeniero electrónico de la Universidad Santo Tomás y graduado con honores por su trabajo de grado en aprendizaje de máquina con algoritmo genético. Ha sido docente en áreas de la inteligencia artificial (IA) desde 2003 e investigador en IA bio-inspirada desde 2008. Docente en la Corporación Unificada Nacional de Educación Superior (CUN). Contacto: dante_sterpin@cun.edu.co
} 


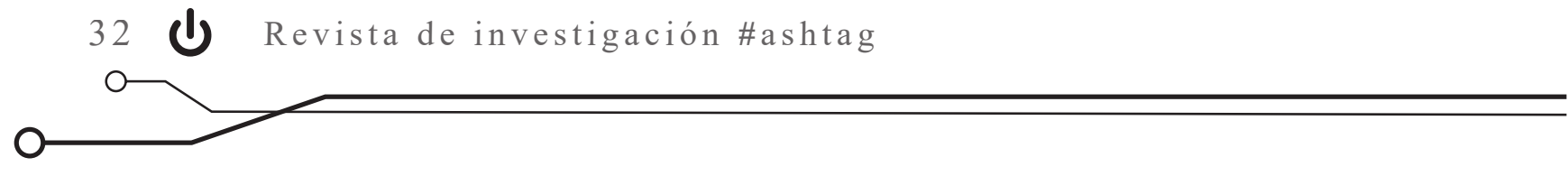

\section{Introducción}

En el contexto de la inteligencia computacional existen diferentes métodos bioinspirados orientados a resolver problemas complejos, en los que los métodos convencionales no sirven o son inaplicables (Maldonado y Gómez, 2010). La inteligencia artificial clásica da solución a ciertos problemas de optimización mediante heurísticas, mientras que la vida artificial lo hace al emplear metaheurísticas, como metodologías para el diseño de heurísticas (Talbi, 2009; Maldonado y Gómez, 2010) o con el fin de seleccionar

\section{Darwinismo universal}

Hablar de evolución biológica es prácticamente referenciar la teoría darwiniana que es, probablemente, la única teoría que puede explicar el fenómeno de la vida (Dawkins, 1983). La evolución solo debe exhibir tres principios fundamentales: herencia, variación y eficacia. El primero se define mediante la correlación entre las características de los progenitores y las de sus descendientes; el segundo implica generar individuos con características diferentes a través de imprecisiones en la herencia; mientras que el último es el grado de idoneidad para la supervivencia y la reproducción (Lewontin, 1970; Aunger, 2002).

\section{Lamarckismo y efecto Baldwin}

Históricamente, el lamarckismo surgió antes del darwinismo, pero la evidencia en su contra lo establece como un error científico, debido a su imposibilidad biológica. Hay dos principios lamarckianos: la herencia genética de las características aprendidas en el tiempo-de-vida y la evolución mediante el uso y desuso (Dawkins, heurísticas o metaheurísticas en problemas de complejidad creciente (Maldonado y Gómez, 2010; Burke, Gendreau, Hyde, Kendall, Ochoa, Özcan, y Qu, 2013). Las metaheurísticas pueden ser de solución única o múltiple, emplean poblaciones de soluciones y entre sus exponentes están los algoritmos evolutivos inspirados en la evolución biológica fundamentada en la selección, recombinación y mutación de genes (Goldberg, 1989; Talbi, 2009).

El darwinismo clásico está restringido a la materia orgánica y fue generalizado por Lewontin (1970) y Dawkins (1983), de tal forma que resulta aplicable a cualquier proceso de carácter evolutivo (Aunger, 2002). Ambas generalizaciones identifican ciertas unidades sobre las cuales se ejerce la presión selectiva. En el darwinismo universal de Dawkins: 1) dichas unidades son denominadas replicadores (Dawkins, 1976); 2) se considera que cualquier proceso evolutivo se basa en la respectiva dinámica de replicación, y 3) la naturaleza de los replicadores no está restringida a la biología orgánica (Dawkins, 1983; Aunger, 2002).

1986). El ejemplo clásico del lamarckismo es el cuello de la jirafa: este supone que las jirafas primitivas no tenían cuello largo, pero por la necesidad de comer las hojas altas de los árboles se esforzaron en usar su cuello, estirándolo, hasta lograr su versión actual. 
A finales del siglo XIX se demostró que los cambios corporales adquiridos no alteran las células sexuales (Aunger, 2002) y, a pesar de dicho error, puede afirmarse que el aprendizaje en el tiempo-de-vida sí influye favorablemente en la evolución; esto se denomina como el efecto
Baldwin (Lamma, Riguzzi y Pereira, 2003). Esta postura considera que las adaptaciones aprendidas en el tiempo-de-vida pueden incrementar la probabilidad de supervivencia de un individuo, dándole ventaja a los genes garantes de dichas capacidades adaptativas.

\section{Hipótesis memética de la evolución cultural}

Entre los replicadores no-genéticos se tiene a los memes, concebidos originalmente por Richard Dawkins (1976) como unidades de transmisión cultural con la capacidad de 'saltar' entre cerebros a través de la imitación. En esta concepción, los memes pueden ser canciones, ideas científicas, prendas de vestir, creencias religiosas o artefactos tecnológicos y tienen una mayor perpetuidad en comparación con sus homólogos, los genes (Dawkins, 1976). Adicionalmente, los memes han sido vistos como libros, recetas, pinturas y partituras (Blackmore, 1999); además, se han analizado como parásitos (Aunger, 2002) y como virus mentales (Dawkins, 1993; Brodie, 2009). El estudio de estos replicadores hipotéticos está orientado a explicar la evolución cultural desde el darwinismo (Dawkins, 1976; Blackmore, 1999; Aunger, 2002) y define una ciencia denominada memética, cuyos principales paladines han sido Richard Dawkins, Susan Blackmore y Daniel Dennett.

\section{Naturaleza neuronal de los memes}

Frente a ese 'libertinaje del sustrato' entre los meméticos, Aunger (2002) establece que los memes solo pueden estar en el cerebro y, por lo tanto, el sustrato material que los soporta está en la composición cerebral. Así, los replicadores meméticos son, con mayor precisión, replicadores neuromeméticos o neuromemes, y solo
Como ciencia, la memética se encuentra en un estado semejante al de la genética en el momento en que Gregor Mendel presentó su trabajo en 1865, pues en esa época se desconocían la estructura molecular del ADN y el proceso por el cual se heredan las características biológicas. Actualmente, la herencia cultural basada en memes es hipotética, pues aún se desconoce la composición material de los memes y su mecanismo de replicación. Al respecto, se tienen conjeturas muy amplias y diversas: Dawkins (1976) los ubica en el cerebro y en artefactos como libros y computadores; Blackmore (1999) los incluye en el comportamiento y sus productos; mientras que Dennett (2006) establece que los memes son mera información independiente del sustrato material que les de soporte. Robert Aunger (2002) considera que dicha diversidad memética viola el principio por el que se establece que un replicador solo puede tener un único sustrato material $\mathrm{y}$, por esto, resulta complicado ubicar a los memes.

pueden existir como estados en nodos neuronales, que pueden ser neuronas individuales, redes de neuronas o las conexiones sinápticas entre ellas. Cuando dichos nodos generan patrones de disparo neuronal, intervienen en otros procesos neuronales o implican movimientos musculares, con los que envían señales sociales al medio en 


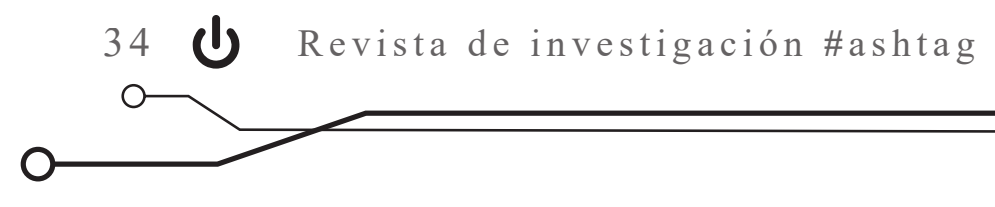

forma de comportamiento o en forma de comunicación verbal y no-verbal.

En contraste, con los memes convencionalmente intangibles, los neuromemes sí cumplen con los cuatro principios fundamentales de un replicador: causación, similaridad, transferencia de información y duplicación. Esto implica, entonces, que los memes no están presentes en los comportamientos -en ninguna otra señal social, y tampoco están en los artefactos- ni en ningún otro producto cultural. Aunger (2002) emplea varias instancias culturales para soportar esta última afirmación y, así, al atender la cuestión del libertinaje del sustrato, deja de dispersarse la ubicación de los memes, asumiéndolos exclusivamente ubicados en el cerebro, en el que

\section{Sociedades artificiales}

Las metaheurísticas basadas en poblaciones, como herramientas de modelamiento y simulación de sistemas complejos, también sirven para modelar dinámicas naturales y sociales, con "la posibilidad de juzgar, probar y validar, mediante simulaciones computacionales, hipótesis, conjeturas y teorías sobre los sistemas naturales" (Maldonado y Gómez, 2010, p. 21). Al tener agentes situados, autónomos, proactivos y sociales, comunicándose e interactuando entre ellos, se tienen sistemas multiagente capaces de resolver problemas complejos (Ferber, 1999; Wooldridge, 2009), o sociedades artificiales que simulan fenómenos sociales (Sawyer, 2003; González, 2004; Squazzoni, Jager y Edmonds, 2014).

En el programa de Ingeniería Electrónica de la CuN, el autor de este artículo ha estado investigando sobre cómo lograr un modelo computacional basado en sociedades artificiales, capaz de ayudar en el estudio de fenómenos sociales son capaces de replicar sus patrones de disparo neuronal.

Aunger (2002) conjetura que la replicación neuromemética pudo haberse originado intracerebralmente y que cuando los neuromemes lograron emitir señales sociales pudieron replicarse intercerebralmente, lo que corresponde con la imitación. Así, los memes no 'saltan' entre cerebros, pues estas señales no transportan memes, sino apenas la información necesaria para desencadenar la reacción replicativa en un nuevo anfitrión con cerebro. Aunger asume esto último con el respaldo de los módulos lingüísticos de Chomsky y afirma que, de esta forma, los neuromemes se replican sin lamarckismo.

como la moral (Hauser, 2006) y la religión (Dennett, 2006), además de abordar posibles soluciones a algunas de sus problemáticas, como la doble-moral (Randsley de Moura y Abrams, 2013; Zaikman y Marks, 2014) y el fanatismo religioso (Brodie, 2009), por ejemplo. Para dicho modelo se ha considerado que sería más apropiado permitir que la complejidad cultural implicada emerja de la interacción entre replicadores culturales simples. De esta manera, ese modelo está orientado a vislumbrar cómo somos parasitados los seres humanos, ya que, así como somos vehículos para la longevidad de nuestros genes (Dawkins, 1976), también somos usados por nuestras ideas y creencias. Adicionalmente, esto ayudaría a caracterizar una ética robótica (Veruggio, Solis y Van der Loos, 2011) al desarrollar un mecanismo moral culturalmente adquirido, para el tipo de robots empleados en el cuidado de adultos mayores (Espingardeiro, 2014). 


\section{Computación evolutiva de tipo memético}

John Holland (1975) propuso los denominados algoritmos genéticos (GA por su sigla en inglés); así, definió las características generales de los algoritmos evolutivos (Hart, Krasnogor y Smith, 2005). En estos hay una población de posibles soluciones a un problema (codificadas genéticamente a manera de individuos); con una función de idoneidad se evalúan las soluciones frente a las exigencias del problema y, al emplear la calificación obtenida, se seleccionan los individuos mejor adaptados, se emparejan y se les aplican los operadores genéticos de cruzamiento y mutación. De este modo, se obtiene una nueva generación de individuos, de forma tal que el ciclo continúa hasta tenerse una solución lo suficientemente eficaz para el problema en cuestión.
Pablo Moscato (1989), al introducir el concepto de meme en el ciclo evolutivo de los GA, originó los algoritmos meméticos (MA por su sigla en inglés). Dicha propuesta generó una gran controversia, ya que, a pesar de considerar características meméticas de la evolución cultural, relega el concepto del replicador memético a un simple operador con el que se logran realizar mejoras locales durante el ciclo evolutivo del GA. De esta manera, se desechan los principios básicos del darwinismo universal aplicado a la evolución cultural a través de memes (Meuth, Lim, Ong y Wunsch, 2009).

A continuación, se explican los tres tipos de MA y otros dos modelos computacionales inspirados en la memética, pero que no derivan de lo propuesto por Moscato.

\section{Algoritmos meméticos de la generación (MAl)}

También denominados GA híbridos o lamarckianos, se caracterizan por combinar el potencial de la búsqueda global propia del GA con alguna técnica de búsqueda local (Hart et al., 2005; Meuth et al., 2009). Esta última es el meme a través del cual cada individuo en la población mejora la solución que representa genéticamente, como una forma de aprendizaje en el tiempo-de-vida. Existen dos mecanismos para incorporar la mejora memética en la población de soluciones, el lamarckiano y el baldwiniano (Hart et al., 2005; Krasnogor, Aragón y Pacheco, 2006; Le, Neri y Ong, 2015): en el primero se recodifica el genotipo individual según el resultado de la mejora, mientras que en el segundo no; ahora bien, en ambos se actualiza el valor de la idoneidad genética individual. Pese a que, en contraste con el efecto Baldwin, el lamarckismo no es natural en absoluto, la ingeniería prefiere esta última perspectiva por su efectividad en la resolución de problemas (Le, Ong, Jin y Sendhoff, 2009).

Durante el ciclo evolutivo de los MA es posible utilizar heurísticas como técnica híbrida en más de un momento. Así, es posible aplicar memes tras el cruzamiento genético $y$, nuevamente, tras la mutación: para mejorar los conjuntos de soluciones previamente conocidas, en lugar de inicializar aleatoriamente la población, vale la pena añadir memes. De igual forma, pueden incorporarse modificaciones en la selección de progenitores y directamente en los operadores genéticos (Hart et al., 2005).

La ventaja de estos algoritmos híbridos se detalla en contraste con el desempeño de los GA frente a algún problema típico de optimización. Estos últimos localizan con rapidez la zona óptima, 


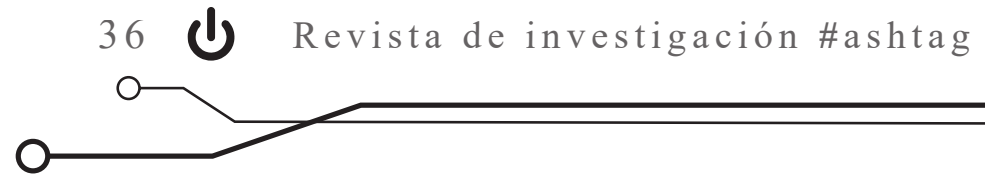

aunque son lentos convergiendo al punto óptimo. Por este motivo, para Hart et al. (2005), los GA son buenos explorando ampliamente el espacio de búsqueda, pero no tanto en la explotación de las zonas prometedoras. Con una búsqueda local, como el ascenso-de-colina o el descenso-del-gradiente, los MA exploran estas zonas y, así, convergen más rápido. Sin embargo, la literatura muestra que no es necesario emplear el concepto de meme en algoritmos híbridos con búsqueda global y local (Radetic, Pelikan y Goldberg et, julio del 2009).

\section{Algoritmos meméticos de 2a generación (MA2)}

En este grupo se incluyen los MA dotados con un conjunto de varias búsquedas locales disponibles para ser adaptativamente escogidas, según las mejorías obtenidas. En la literatura suelen mencionarse tres tipos de MA en este nivel: multimeme, hiperheurísticos y metalamarckianos (Ong, Lim, Zhu y Wong, 2006; Meuth et al., 2009; Le et al., 2015). En contraste con los MA1, los MA2 pueden seleccionar los memes que aplican en su ciclo evolutivo.

En los algoritmos multimeme, los memes se codifican como parte del genotipo y su respectiva decodificación es empleada para el aprendizaje del individuo: el material memético se hereda genéticamente de progenitores a sucesores. De otro lado, en los algoritmos hiperheurísticos y metalamarckianos, hay un conjunto de memes recompensados, según su éxito, como técnicas de aprendizaje individual con el fin de ser subsecuentemente replicados para emplearlos en futuras mejoras locales (Meuth et al., 2009).

En los MA hiperheurísticos hay tres categorías para escoger memes: aleatoria, avara y con función de selección. En la primera, se seleccionan al azar y se mantienen mientras no haya otros con mejor desempeño; en la segunda, se prueban todos los memes y se escoge el que tenga mejor desempeño (Ong et al., 2006). Finalmente,
Cowling, Kendall y Soubeiga (2001) y Kendall, Cowling y Soubeiga (2002) presentan una función de selección para evaluar la efectividad de los memes al conocerse la región explorada del espacio de búsqueda (Ong et al., 2006).

En cuanto a la función de selección, el aprendizaje metalamarckiano representa una evolución de los MA hiperheurísticos. Este aprendizaje es una coordinación simple de memes; allí se descompone el espacio de decisiones para optimizar cada subárea por separado, al asumir que cada una necesitaría un meme diferente (Le et al., 2015). El aprendizaje metalamarckiano implica competición y coordinación de los memes empleados, ya que en cada subárea compiten según su habilidad para explorar su respectiva región en el espacio de búsqueda y, simultáneamente, se coordinan con los mejores en las otras subáreas para encontrar la solución global (Ong et al., 2006). Los MA hiperheurísticosy metalamarckianos evidencian propagación memética no-genética junto con el método de difusión de Nguyen, Ong y Lim, (julio del 2008). En este último trabajo, entre otros (Ullah, Sarker, Comfort y Lokan, 2007; Huy, Soon, Hiot y Krasnogor, 2009), se utiliza una población de agentes organizados en cuadrícula, tal como en los autómatas celulares empleados en simulación social (González, 2004). 


\section{Algoritmos meméticos de 3a generación (MA3)}

En este último grupo están los MA basados en los paradigmas de coevaluación y autogeneración. A diferencia de los MA2, acá el conjunto de memes a escoger no se conoce con antelación; la búsqueda local se coadapta con las posibles soluciones (Meuth et al., 2009), a través de un ciclo evolutivo memético alterno al ciclo genético convencional. De esta forma, los MA3 implican la evolución del tipo de memes que tan solo existen y se utilizan en los MA1 o son seleccionados y heredados en los MA2. Esta es la diferencia esencial entre los tres grupos de MA, en virtud de los principios fundamentales del darwinismo universal (Nguyen et al., 2008).

Smith (2003) presenta los mecanismos implicados en el ciclo evolutivo en el que evolucionan los memes, el operador de búsqueda local (LSO por su sigla en inglés), en los MA coevolutivos. En este ciclo evolutivo los memes son representados, inicializados, evaluados, recombinados y

\section{Sistemas multiagente meméticos (Memas)}

Al considerar el papel, apenas complementario, de los memes en los MA y con la intención de tener un modelo meme-céntrico, Pan, Feng, Ong, Kang, Tan y Miao (2010) introdujeron la composición del autómata memético (Mema) y Chen, Ong, Lim y Tan (2011) la formalizaron conceptualmente como un modelo capaz de acoger una gran variedad de herramientas de representación de conocimiento, como representaciones meméticas, además de estar conformado por los tres mecanismos darwinianos de la evolución memética: selección, propagación y variación.

Pan et al. (2010) y Feng, Ong, Tan y Chen (junio del 2011) presentan sistemas multiagente meméticos (Memas) basados en la conceptualización modificados. Krasnogor y Gustafson (2002) presentaron el paradigma autogenerativo como una especie de MA "verdadero", resaltando que, de esta forma, se descubren autónomamente nuevas estrategias de búsqueda local que evolucionan con las soluciones que mejoran al aplicarlas, de forma que se evite perder tiempo refinando soluciones ya cercanas al óptimo. En este último trabajo, el doble ciclo evolutivo está conformado por dos poblaciones, una genético-lamarckiana y otra memética, en la que los memes modifican a los fenotipos y, mediante enseñanza, por ejemplo, los fenotipos pueden modificar a los memes. En estos MA autogenerativos, los memes especifican reglas, programas, heurísticas, conductas o LSO. Pero, además de ser simples refinadores locales, también son considerados como fuente de bloques constructivos (Krasnogor y Gustafson, 2004) semejantes a los esquemas genéticos presentes en la teoría de los GA (Goldberg, 1989).

del Mema. En estos, cada agente está dotado con una red neuronal auto organizativa con aprendizaje por refuerzo, denominada TD-Falcon (Tan, Lu y Xiao, 2008). Feng et al. (2011) diagraman explícitamente las dos formas de evolución memética: la intraagente, en términos de aprendizaje individual, y la interargente, en términos de aprendizaje cultural. La primera es un mecanismo de Q-Learning (Sutton y Barto, 1998) que no está inspirada en el darwnismo, pero acarrea los conceptos de exploración y explotación propios de los algoritmos evolutivos (Hart et al., 2005). La segunda implica un protocolo a través del cual un agente solicita ayuda a otros para imitar su comportamiento al incorporar el respetivo meme. 


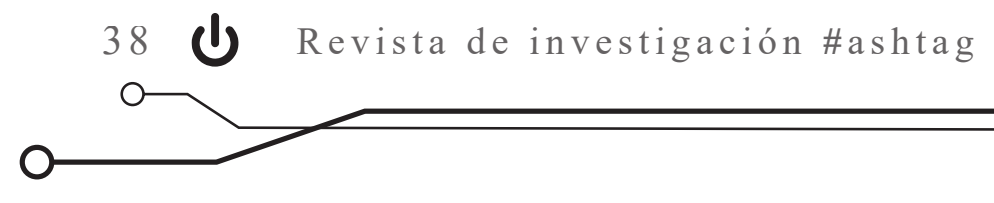

Al usar TD-Falcon como neuroagente, los memes en el Memas corresponden a los nodos almacenados en la segunda capa de dicha red neuronal, lo cual es un mapeo de estado-acción-recompensa asimilado por el agente en su tiempo-devida (Feng et al., junio del 2011). El conjunto de estos nodos en cada agente configura su experiencia en la solución de un problema; esto corresponde con la interpretación de los memes como bloques constructivos en el Mema: con esto, se trasciende el paradigma de las estrategias para el refinamiento local en los MA.

\section{Modelo de herencia dual gene-meme (DIM)}

Este modelo surge como contrapropuesta a diferentes modelos evolutivos con una serie de falencias, entre ellos, los basados en agentes, con propagación horizontal, con desarrollo fenotípico (lamarckiano o no), además del tipo de sistemas evolutivos basados en redes neuronales con aprendizaje social (Sernas). Estas falencias se analizaron con respecto a la capacidad de modelar la interacción entre lo genético y lo cultural al: 1) considerar la concepción memética de Dawkins; 2) notar que, desde el diseño original de Moscato, los algoritmos meméticos no incorporan la evolución conjunta de genes y memes, y 3) objetar el uso de redes neuronales en los Sernas por la dependencia de agentes expertos iniciales, la típica demora en su entrenamiento con descenso-del-gradiente y la imprecisión resultante de su proceso de aprendizaje. Pese a esto último, el modelo de herencia dual (DIM, por su sigla en inglés) rescata de los Sernas el uso de dos contenedores separados para genes y memes y considera el uso de agentes simples con una estrecha relación gene-meme, para garantizar que el modelo posea características filogenéticas, ontogenéticas y sociogenéticas.
Lo anterior ocurre en la representación memética intraagente, ya que, en cuanto a la representación interargente, se tiene la expresión memética en forma de comportamiento. Con el empleo de TD-Falcon, la selección memética es medida por cuánto se prefiera de un meme, según sea capaz de lograr cierta recompensa, en términos del Q-Learning (Sutton y Barto, 1998). El Mema también tiene transmisión memética vertical y horizontal (Gong, 2010) por medio de la propagación de memes vía imitación, y permite, adicionalmente, la respectiva variación memética.

Como característica particular, el DIM es capaz de evidenciar la divergencia que puede darse entre la evolución genética y la cultural. Los ejemplos clásicos de esta divergencia son el celibato sacerdotal y el suicidio religioso, ya que sus propósitos culturales contradicen los propósitos genéticos de procreación y preservación de la vida biológica.

La validación del DIM se realizó usando agentes orientados al foraging en una red aleatoria. Los genes codifican posibles rutas a través de dicha red, mientras los memes se desarrollan en el momento del nacimiento, copiando segmentos del genoma en el memoma. Estos segmentos fueron considerados como complejos meméticos o "memplejos" (Dawkins, 1976). Así, se almacenan varias rutas-solución en el memoma; con alguno de estos memeplejos los agentes evidencian un determinado comportamiento, con el fin de obtener la información fenotípica necesaria para la selección. En el DIM se garantiza que el genoma permanece inerte durante el tiempo-de-vida del agente, mientras que el memoma se modifica con aprendizaje individual, en la interacción 
agente-ambiente y con aprendizaje social en la interacción con otros agentes. El DIM considera que la propagación de genes es exclusivamente vertical, mientras que la propagación de memes puede ser vertical, horizontal y diagonal (Gong, 2010).

\section{Computación evolutiva cultural sin memes}

En esta sección se revisan algunos modelos computacionales de tipo evolutivo-cultural que no se fundamentan en los conceptos de la memética,

\section{Algoritmos culturales}

Los MA también han sido nombrados algoritmos (Nguyen et al., julio del 2008; Huy et al., 2009), entre ellos, uno inspirado en la cultura que se denomina algoritmo cultural (CA por su sigla en inglés). Los CA fueron propuestos por Robert Reynolds (1994) como una variación del GA, al utilizar el teorema de los esquemas genéticos (Goldberg, 1989), a manera de benchmark, para investigar los motivos por los que la evolución cultural es más acelerada que la evolución genética. Estos cA se caracterizan por combinar un espacio poblacional con un espacio de conocimiento cultural, que

\section{Aprendizaje cultural con redes neuronales}

El aprendizaje por imitación es considerado como la capacidad cognitiva que permite la herencia y evolución cultural en las sociedades humanas (Dawkins, 1976). Dicha capacidad se atribuye a las neuronas-espejo que están presentes en el cerebro de los homínidos. Estas neuronas se activan cuando un individuo ejecuta una acción y observa a otro realizando esa misma acción, por lo que se considera que dichas neuronas tienen la función de relacionar la acción observada con el patrón motriz necesario para imitarla (Borenstein y Ruppin, 2004). Sin embargo, Aunger (2002) establece que esta perspectiva imitativa no es apropiada y que en su lugar pero se inspiran en la adquisición y uso de conocimiento cultural a través del aprendizaje social y la imitación.

puede ser de tipo normativo, situacional, de dominio, histórico o topográfico (Reynolds y Peng, 2005). La población en los CA posee las mismas características y dinámicas presentes en los GA, es decir, es inicializada, sus individuos son evaluados con cierta función objetivo y los mejores son seleccionados para aplicarles operadores genéticos. Adicionalmente, en los cA se aprovecha la experiencia poblacional a través del aprendizaje de ciertas creencias que son generalizadas, para conformar el respectivo conocimiento cultural y modificar el desempeño individual.

sucede la replicación de neuromemes. Por su parte, Borenstein y Ruppin emplearon un GA con una población de neuroagentes, para mostrar el desarrollo de la capacidad de imitación bajo la perspectiva de las neuronas-espejo.

Según Curran y O’Riordan (2007), hay un híbrido entre el aprendizaje poblacional de un GA, el individual de los neuroagentes en la población y otro de tipo cultural. Para este último tipo de aprendizaje, con el fin de comunicar sus verbalizaciones sobre el medio ambiente a pupilos con la capacidad de imitarlas, los mejores individuos en cada generación toman el rol de tutores. Con 


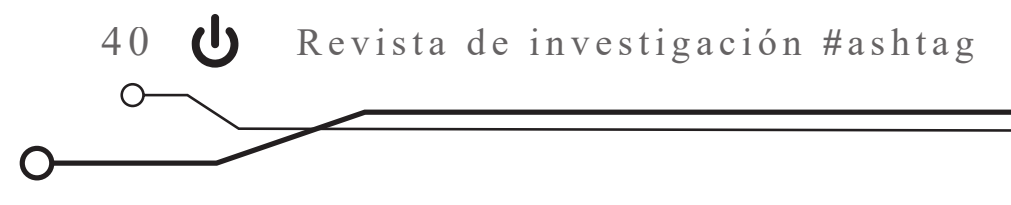

esta interacción entre tutores y pupilos, toda la población desarrolla autónomamente un léxico neuronalmente codificado y acelera su propia

\section{Aprendizaje cultural con robots móviles}

Un algoritmo evolutivo puede verse un poco más natural al combinarse con robots móviles en entornos reales. Así, en el campo de la robótica evolutiva, suelen emplearse algunas metaheurísticas para la evolución de estructuras robóticas y controladores para robots móviles (Nedjah, Coello y Mourelle, 2007; Floreano, Husbands y Nolfi, 2008). Al detallar las revisiones recientes puede notarse que, al parecer, en este campo nunca se ha empleado el tipo de metaheurísticas propias de la computación memética (Bongard, 2013; Doncieux, Bredeche, Mouret y Eiben, 2015). No obstante, Heinerman, Rango y Eiben (2015) hacen uso de cierto memoma capaz de evolucionar junto con un genoma destinado a habilitar/deshabilitar los sensores de un robot y, con aprendizaje individual y social, varios robots conforman un conocimiento cultural en dicho memoma. Todo esto último se abordó sin hacer referencia alguna a los conceptos de la evolución cultural desde el punto de vista de la memética, aun cuando dicho memoma alude al concepto de memotipo, es decir, el conglomerado de los

\section{Conclusión}

En esta revisión se detallaron las características fundamentales de los algoritmos meméticos (MA1, MA2, MA3) y de los culturales (CA), además de los sistemas multiagentes meméticos (Memas), de algunos casos de aprendizaje cultural artificial y del DIM: un modelo de herencia dual genética y memética. En el caso de los MA y los CA, es evidente que la finalidad de lo cultural es acelerar y mejorar el desempeño de los evolución, mostrando así el papel del lenguaje y la educación en la cultura.

memes propios de una cultura, análogo al concepto de genotipo como conglomerado de los genes propios de una especie (Dawkins, 1976; Blackmore, 1999).

En Acerbi y Marocco (2009) se combinaron la herencia genética, el aprendizaje social y neurocontrol de robots móviles, con el fin de realizar una coevolución no-lamarckiana entre genes y comportamiento. En este último trabajo se deseaba estudiar el efecto de explotar cierta 'socialidad' natural, genéticamente codificada, para orientar el aprendizaje individual en pro de incrementar la supervivencia en una sociedad de robots que forrajean en un ambiente desconocido. Al respecto, resulta cuestionable que la socialidad, como tendencia natural a ser parte de un grupo social (Pam, 13 de abril del 2013), pueda codificarse genéticamente con un único bit, pues así dichos autores asumieron que esta tendencia puede permutarse entre sí-social y no-social, para que un robot se comportara exclusivamente como lo uno o lo otro.

algoritmos genéticos (GA); por otro lado, en los $\mathrm{MA}$, la relación entre genes y memes no es natural, sus memes son tan solo aprendizajes individuales en procura de mejorar, con lamarckismo frecuentemente, el genotipo de una población. En el caso de los Memas hay una ausencia total de elementos genéticos, hecho que implica las dificultades conceptuales que Aunger (2002) encuentra en la memética. Por su parte, los modelos 
de aprendizaje cultural acá detallados, y en especial el DIM, tienen elementos más cercanos a la naturaleza; pero, aun así, resultan insuficientes para modelar la evolución cultural basada en la neuromemética. Frente a esta problemática, el autor de este artículo ha estado desarrollando un sistema multiagente para la coevolución de genes y neuromemes en una sociedad de robots. Para esto: 1) establece que lo genético debe codificar una maquinaria corpóreo-mental capaz de sustentar lo neuromemético; 2) reduce el uso de redes neuronales artificiales, y 3) minimiza el tiempo necesario para el respectivo aprendizaje cultural.

\section{Referencias}

Acerbi, A. y Marocco, D. (Junio del 2009). Orienting learning by exploiting sociality: An evolutionary robotics simulation. Conferencia presentada en 2009 International Joint Conference on Neural Networks, Atlanta, Estados Unidos, 20-27. DOI: https://doi.org/10.1109/ IJCNN.2009.5178607

Aunger, R. (2002). The Electric Meme: A New Theory of How We Think. Nueva York: Free Press.

Blackmore, S. (1999). The Meme Machine. Oxford: Oxford University Press.

Bongard, J. (2013). Evolutionary robotics. Communications of the ACM, 56(8), 74-83.

Borenstein, E. y Ruppin E. (2004). Envolving imitating agents and the emergence of a neural mirror system. En M. Bedau, P. Husbands, T. Ikegami, J. Pollack y R. Watson (eds.), Artificial life IX. Proceedings of the Ninth International Conference on the Simulation and Synthesis of Living Systems (pp. 146-151). Cambridge, Estados Unidos: The MIT press.

Brodie, R. (2009). Virus of the Mind: The New Science of the Meme. Nueva York: Hay House.

Burke, E., Gendreau, M., Hyde, M., Kendall, G., Ochoa, G., Özcan, E. y Qu, R. (2013). Hyper-heuristics: a survey of the state of the art. Journal of the Operational Research Society, 64(12), 16951724. DOI: https:// doi.org/10.1057/jors.2013.71

Chen, X., Ong, Y., Lim, M. y Tan, K. (2011). A multi-facet survey on memetic computation. IEEE Transactions on evolutionary computation, 15(5), 591-607. DOI: https://doi.org/10.1109/ TEVC.2011.2132725

Cowling, P., Kendall, G. y Soubeiga, E. (2001). A Hyperheuristic Approach to Scheduling a Sales Summit. En E. Burke y W. Erben (eds.), Practice and Theory of Automated Timetabling III. PATAT 2000. Lecture Notes in Computer Science (vol. 2079) (pp. 176-190), Konstanz, Germany: Springer. 


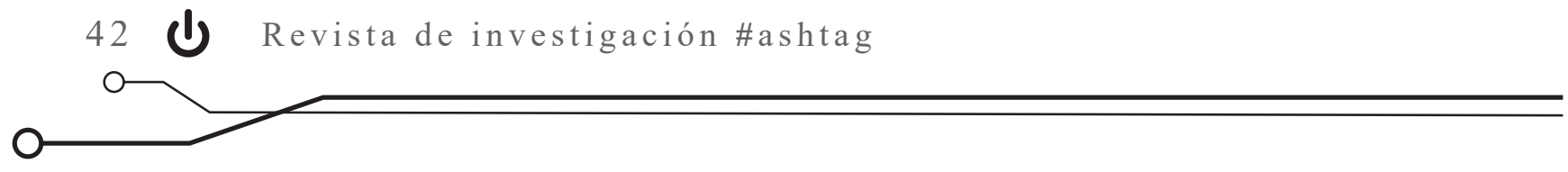

Curran, D. y O'Riordan, C. (2007). The effects of cultural learning in populations of neural networks. Artificial Life, 13(1), 45-67. DOI: https:/ / doi.org/10.1162/artl.2007.13.1.45

Dawkins, R. (1976). The selfish gene. Oxford: Oxford University Press.

Dawkins, R. (1983). Universal Darwinism. En D. Bendall (ed.), Evolution from Molecules to Man (pp. 403-425). Nueva York: Cambridge University Press.

Dawkins, R. (1986). The Blind Watchmaker: Why the Evidence of Evolution Reveals a Universe Without Design. Nueva York y Londres: WW Norton \& Company.

Dawkins, R. (1993). Viruses of the mind. En B. Dahlbom (ed.), Dennett and His Critics: Demystifying Mind (pp. 13-27). Oxford: Blackwell.

Dennett, D. (2006). Breaking the Spell: Religion as a Natural Phenomenon. Estados Unidos Penguin Books.

Doncieux, S., Bredeche, N., Mouret, J-B. y Eiben A. (2015). Evolutionary robotics: what, why, and where to. Frontiers in Robotics and AI, 2(4), 1-18. DOI: https://doi.org/10.3389/ frobt.2015.00004

Espingardeiro, A. (2014). A roboethics framework for the development and introduction of social assistive robots in elderly care (tesis de doctorado). Universidad de Salford, Manchester. Recuperado de https://bit.ly/2toFxNQ

Feng, L., Ong, Y., Tan, A. y Chen, X. (Junio del 2011). Towards human-like social multi agents with memetic automaton. Conferencia presentada en Congress of Evolutionary Computation (CEC), Nueva Orleans, Estados Unidos, 1092-1099. DOI: https://doi.org/10.1109/ CEC.2011.5949739

Ferber, J. (1999). Multi-Agent System: An Introduction to Distributed Artificial Intelligence. Boston: Addison-Wesley Longman Publishing Co.

Floreano, D., Husbands, P. y Nolfi, S. (2008). Evolutionary robotics. En B. Siciliano y O. Khatib (eds.), Springer handbook of robotics (pp. 1423-1451). Berlin: Springer.

Goldberg, D. (1989). Genetic algorithms in search, optimization, and machine learning. Boston: Addison-Wesley Longman Publishing Co.

Gong, T. (2010). Exploring the roles of horizontal, vertical, and oblique transmissions in language evolution. Adaptive Behavior, 18(3-4), 356-376.

González, S. (2004). ¿Sociedades artificiales? Una introducción a la simulación social. Revista Internacional de Sociología, 62(39), 199-222. Recuperado de https:/ / bit.ly/2PpCa1y 
Hart, W., Krasnogor, N. y Smith, J. (2005). Memetic evolutionary algorithms. En W. Hart, N. Krasnogor y J. Smith. (eds.), Recent Advances in Memetic Algorithms (pp. 3-27). Berlin; Heidelberg; New York: Springer.

Hauser, M. (2006). Moral Minds: How Nature Designed our Universal Sense of Right and Wrong. Nueva York: Harper Collins.

Heinerman, J., Rango, M. y Eiben, A. (Diciembre del 2015). Evolution, individual learning, and social learning in a swarm of real robots. Conferencia presentada en 2015 IEEE Symposium Series on Computational Intelligence, Cape Town, Sur Africa, 1055-1062. Dor: https://doi. org/10.1109/SSCI.2015.152

Holland, J. (1975). Adaptation in Natural and Artificial Systems. An Introductory Analysis with Applications to Biology, Control, and Artificial Intelligence. Míchigan: University of Michigan Press.

Huy, N., Soon, O., Hiot, L. y Krasnogor, N. (2009). Adaptive cellular memetic algorithms. Evolutionary Computation, 17(2), 231-256. Dor: https://doi.org/10.1162/evco.2009.17.2.231

Kendall, G., Cowling, P. y Soubeiga, E. (2002). Choice function and random hyperheuristics. Conferencia presentada en 4th Asia-Pacific Conference on Simulated Evolution and Learning, Singapore, 667-671. Recuperado de https:/ / bit.ly/2PSE9dU

Krasnogor, N., Aragón, A. y Pacheco, J. (2006). Memetic algorithms. En E. Alba y R. Martí (eds.), Metaheuristic Procedures for Training Neutral Networks (pp. 225-248). Boston: Springer. DoI: https:// doi.org/10.1007/0-387-33416-5

Krasnogor, N. y Gustafson, S. (2002). Toward truly "memetic" memetic algorithms: Discussion and proofs of concept. En D. Corne, G. Fogel, W. Hart, J. Knowles, N. Krasnogor, R. Roy, J. Smith y A. Tiwari (eds.), Advances in Nature-Inspired Computation: The PPSN VII Workshops, (pp. 9-10). Reading: PEDAL; University of Reading.

Krasnogor, N. y Gustafson, S. (2004). A study on the use of "self-generation" in memetic algorithms. Natural Computing, 3(1), 53-76. DoI: https:// doi.org/10.1023/B:NACO.0000023419.83147.67

Krasnogor, N. y Smith, J. (2005). A tutorial for competent memetic algorithms: models, taxonomy and design issues. IEEE Trans Evol Comput, 9, 474-488.

Lamma, E., Riguzzi, F. y Pereira, L. (2003). Belief revision via lamarckian evolution. New Generation Computing, 21(3), 247-275. DOI: https://doi.org/10.1007/BF03037475

Le, M., Neri, F. y Ong, Y. (2015). Memetic algorithms. En H. Ishibuchi (ed.), Encyclopedia of Life Support Systems: Computational Intelligence (vol. 2), (pp. 57-86). Singapur: Unesco; Eolss Publishers. 


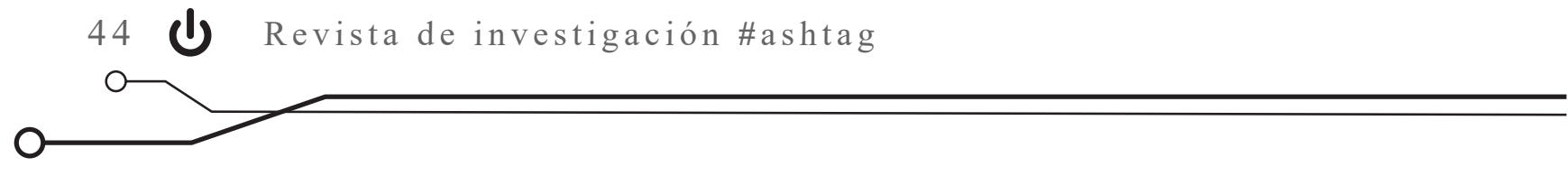

Le, M., Ong, Y., Jin, Y. y Sendhoff, B. (2009). Lamarckian memetic algorithms: Local optimum and connectivity structure analysis. Memetic Computing, 1(3), 175-190. DoI: https://doi. org/10.1007/s12293-009-0016-9

Lewontin, R. (1970). The Units of Selection. Annual Review of Ecology and Systematics, 1, 1-18.

Maldonado, C. y Gómez, N. (2010). Modelamiento y simulación de sistemas complejos. Bogotá: Editorial Universidad del Rosario. Recuperado de https:// bit.ly/36JK4sD

Meuth, R., Lim, M., Ong, Y. y Wunsch, D. (2009). A proposition on memes and meta-memes in computing for higher-order learning. Memetic Computing, 1(2), 85-100.

Moscato, P. (1989). On evolution, search, optimization, genetic algorithms and martial arts: Towards memetic algorithms. Pasadena, Estados Unidos: Caltech. Recuperado de https://bit. ly/38K6hsb

Nedjah, N., Coelho, L. y Mourelle, L. (Eds.). (2007). Mobile robots: The evolutionary approach - Studies in Computational Intelligence (vol. 50). Berlin: Springer.

Nguyen, Q., Ong, Y. y Lim, M. (Julio del 2008). Non-genetic transmission of memes by diffusion. Conferencia presentada en 10th annual conference on Genetic and evolutionary computation, Atlanta, Estados Unidos, 1017-1024. DOI: http:/ / doi.acm.org/10.1145/1389095.1389285

Ong, Y., Lim, M., Zhu, N. y Wong, K. (2006). Classification of adaptive memetic algorithms: A comparative study. IEEE Transactions on Systems, Man, and Cybernetics - Part B (Cybernetics), 36(1), 141-152.

Pam, N. (13 de abril del 2013). Sociality [recurso en línea]. Recuperado de https:/ / bit.ly/34vpt9Q

Pan, Z., Feng, L., Ong, Y., Kang, Y., Tan, A. y Miao, C. (Septiembre del 2010). Meme selection, variation, and transmission in multi-agent system. Conferencia presentada en World Automation Congress, Kobe, Japón, 1-6. Recuperado de https:/ / bit.ly/2rXpo1j

Radetic, E., Pelikan, M. y Goldberg, D. (Julio del 2009). Effects of a deterministic hill climber on hBOA. Conferencia presentada en 11th Annual conference on Genetic and evolutionary computation, Montreal, Canadá, 437-444. DoI: http:// doi.acm.org/10.1145/1830483.1830543

Randsley de Moura, G. y Abrams, D. (2013). Bribery, Blackmail, and the Double Standard for Leader Transgressions. Group Dynamics: Theory, Research, and Practice, 17(1), 43-52. DoI: https://doi.org/10.1037/a0031287 
Reynolds, R. (1994). An introduction to cultural algorithms. En A. Sebald y L. Fogel (eds.), Proceedings of the third annual conference on evolutionary programming (pp. 131-139). River Edge, Estados Unidos: World Scientific Programming.

Reynolds, R. y Peng, B. (2005). Cultural algorithms: computational modeling of how cultures learn to solve problems: an engineering example. Cybernetics and Systems: An International Journal, 36(8), 753-771. DoI: https:// doi.org/10.1080/01969720500306147

Sawyer, R. (2003). Artificial Societies: Multiagent Systems and the Micro-Macro Link in Sociological Theory. Sociological Methods \& research, 31(3), 325-363. DoI: https://doi. org/10.1177/0049124102239079

Smith, J. (Diciembre de 2003). Co-evolving memetic algorithms: A learning approach to robust scalable optimisation. Conferencia presentada en The 2003 Congress on Evolutionary Computation, CEC «03, Canberra, Australia, 498-505. DOI: https://doi.org/10.1109/CEC.2003.1299617

Squazzoni, F., Jager, W. y Edmonds, B. (2014). Social Simulation in the Social Sciences: A Brief Overview. Social Science Computer Review, 32(3), 279-294. DoI: https://doi. org $/ 10.1177 / 0894439313512975$

Sutton, R. y Barto, A. (1998). Reinforcement learning: An introduction. Cambridge, Estados Unidos: MIT Press.

Talbi, E. (2009). Metaheuristics: from design to implementation. Nueva Jersey: John Wiley \& Sons.

Tan, A., Lu, N. y Xiao, D. (2008). Integrating temporal difference methods and self-organizing neural networks for reinforcement learning with delayed evaluative feedback. IEEE Transactions on Neural Networks, 19(2), 230-244. DoI: https:/ / doi.org/10.1109/TNN.2007.905839

Ullah, A., Sarker, R., Comfort, D. y Lokan, C. (Septiembre del 2007). An agent-based memetic algorithm (AMA) for solving constrained optimization problems. Conferencia presentada en 2007 IEEE Congress on Evolutionary Computation, Singapur, Singapur, 999-1006. Dor: https://doi. org/10.1109/CEC.2007.4424579

Veruggio, G., Solis, J. y Van der Loos, M. (2011). Roboethics: Ethics Applied to Robotics. IEEE Robotics \& Automation Magazine, 18(1), 21-22. DoI: 10.1109/MRA.2010.940149

Wooldridge, M. (2009). An introduction to multiagent systems. Glasgow: John Wiley \& Sons.

Zaikman, Y. y Marks, M. (2014). Ambivalent Sexism and the Sexual Double Standard. Sex Roles, 71(9-10), 333-344. 